\title{
A PROBLEM INVOLVING THE $p$-LAPLACIAN OPERATOR
}

\author{
RATAN KR. GIRI AND D. CHOUDHURI
}

Abstract. Using a variational technique we guarantee the existence of a solution to the resonant Lane-Emden problem $-\Delta_{p} u=\lambda|u|^{q-2} u,\left.u\right|_{\partial \Omega}=0$ if and only if a solution to $-\Delta_{p} u=$ $\lambda|u|^{q-2} u+f,\left.u\right|_{\partial \Omega}=0, f \in L^{p^{\prime}}(\Omega)$ ( $p^{\prime}$ being the conjugate of $p$ ), exists for $q \in\left(p, p^{*}\right)$ under certain condition on $\lambda$, where $p^{*}$ is the Sobolev conjugate of $p$.

Mathematics subject classification (2010): 35A15, 35A01.

Keywords and phrases: $p$-Laplacian, elliptic PDE, Palais-Smale condition, Sobolev space.

\section{REFERENCES}

[1] A. Ambrosetti and P. H. Rabinowitz, Dual Variational Methods in Critical Point Theory and Applications, J. Funct. Anal., 14 (1973), 349-381.

[2] B. KAWOHL, Symmetry results for functions yielding best constants in Sobolev-type inequalities, Discrete Contin. Dynam. Systems, 6 (2000), 683-690.

[3] C. GRUmiau AND E. PARINI, On the asymptotics of solutions of the Lane-Emden problem for the p-Laplacian, Archiv der Mathematik, 91, 4 (2008), 354-365.

[4] G. Bachman and L. NARICI, Functional Analysis, Dover Publications, Mineola, New York, 1966.

[5] GREy ERCOLE, On the resonant Lane-Emden problem for the p-Laplacian, Communications in Contemporary Mathematics, 16, 4 (2014), 1350033-1-22.

[6] GREy ERCole, On the resonant Lane-Emden problem for the p-Laplacian, Communications in Contemporary Mathematics, 16, 4 (2014), 1492001-1.

[7] IDRISSA LY, The first eigenvalue for the p-laplacian operator, Journal of inequalities of pure and applied Math., 6, 3 (2005), 1-28.

[8] J. García Azorero And I. Peral Alonso, On limits of solutions of elliptic problems with nearly critical exponent, Comm. Partial Differential Equations, 17 (1992), 2113-2126.

[9] J. I. DÍAZ AND J.E. SAA, Existence et unicité de solutions positives pour certaines équations elliptiques quasilineaires, C. R. Acad. Sci. Paris, 305 (1987), 521-524.

[10] S. Kesavan, Functional Analysis and applications, New age international pvt. Itd., 2003.

[11] L.C. Evans, Partial Differential Equations, Amer. Math. Soc., 2009.

[12] M. F. Bidaut-VÉron, N. Q. Hung And Laurent VÉron, Quaislinear Lane-Emden equations with absorption and measure data, J. Math. Pures Appl., 102, 2 (2014), 315-337.

[13] M. ÔTANI, Existence and nonexistence of nontrivial solutions of some nonlinear degenerate elliptic equations, J. Funct. Anal., 76 (1988), 140-159.

[14] N. Dunford And J. Schwartz, Linear Operators. Part 2: Spectral theory, Wiley (Interscience), New York, 1958.

[15] Nikos E. Mastorakis, Hassan Fathabadi, On the solution of p-Laplacian for non-newtonian fluid flow, W.S.E.A.S. transactions on Math., 8, 6 (2009), 238-245.

[16] P. DRÁBEK, A note on the nonuniqueness for some quasilinear eigenvalue problem, Appl. Math. Lett., 13 (2000), 39-41.

[17] Philippe G. Ciarlet, The finite element method for elliptic problems, Studies in Mathematics and its Applications, Vol. 4, North-Holland Publishing Co., Amsterdam, 1978.

[18] P. Juntinen, P. LindQvist, On the higher eigenvalues for the $\infty$-eigenvalue problem, Calc. Var. partial differential equations, 23 (2005), 169-192. 
[19] Ratan Kr. Giri And D. Choudhuri, Reduced limit approach to semilinear elliptic PDEs with measure data ( arXiv:1605.00870 [math.AP] ).

[20] T. Bhattacharya, E. D. Benedetto and J. Manfredi, Limits as $p \rightarrow \infty$ of $\Delta_{p} u=f$ and related extremal problems, Rend. Sem. Mat. Univ. Politec. Torino, special issue (1991), 15-68. 